\title{
Direct Assessment of Tube Dilation in Entangled Polymers
}

\author{
B. J. Gold, ${ }^{1, *}$ W. Pyckhout-Hintzen, ${ }^{1}$ A. Wischnewski, ${ }^{1}$ A. Radulescu, ${ }^{2}$ M. Monkenbusch, ${ }^{1}$ \\ J. Allgaier, ${ }^{1}$ I. Hoffmann, ${ }^{3}$ D. Parisi, ${ }^{4,5}$ D. Vlassopoulos, ${ }^{4,5}$ and D. Richter ${ }^{1}$ \\ ${ }^{1} J$ Julich Centre for Neutron Science (JCNS) and Institute for Complex Systems (ICS), \\ Forschungszentrum Jülich GmbH, 52428 Jülich, Germany \\ ${ }^{2}$ Jülich Centre for Neutron Science (JCNS) at Maier Leibnitz-Zentrum, \\ Forschungszentrum Jülich GmbH, 85747 Garching, Germany \\ ${ }^{3}$ Institute Laue Langevin (ILL), 3800 Grenoble, France \\ ${ }^{4}$ Institute of Electronic Structure and Laser, FORTH, 70013 Heraklion, Crete, Greece \\ and Department of Materials Science and Technology, University of Crete, 70013 Heraklion, Crete, Greece \\ ${ }^{5}$ Department of Materials Science and Technology, University of Crete, 70013 Heraklion, Crete, Greece
}

(Received 30 September 2018; published 25 February 2019)

\begin{abstract}
A key ingredient within theories focusing on the rheology of entangled polymers is the way how the topological constraints of an entangled chain are lifted by unconstrained segments, i.e., how the constraining tube is dilated. This important question has been addressed by directly measuring the tube diameter $d$ at the scale of the tube by neutron spin echo spectroscopy. The tube diameter $d$ and plateau modulus $G_{N}^{0}$ of highly entangled polyethylene oxide (PEO) chains of volume fraction $c$ that are diluted by low molecular PEO show a concentration dependence $d \propto c^{a / 2}$ and $G_{N}^{0} \propto c^{1+a}$ with an exponent $a$ close to $4 / 3$. This result allows the clear discrimination between different theoretical models that predict $4 / 3$ or other values between 1 and 2 and provides an important ingredient to tube model theories.
\end{abstract}

DOI: $10.1103 /$ PhysRevLett.122.088001

High molecular weight polymer melts and solutions exhibit unique rheological properties such as viscoelasticity with rubberlike behavior at intermediate times and very long terminal relaxation times accompanied by high viscosities, shear thinning, strain hardening, and hierarchical relaxation of branched polymers. They originate from topological constraints that are imposed by the mutually interpenetrating chains. These constraints or entanglements lead to preferred motion in the direction of the chain contour. Much progress in the understanding of polymer chain dynamics was achieved in terms of tube models based on the reptation mechanism [1,2]. The tube thereby is characterized by its diameter $d$ and its contour length $L$. Refinements addressing contour length fluctuations (CLF) of a chain in its tube as well as the effect of constraint release (CR), represent the state of the art. It is important to note, however, that the molecular nature of an entanglement is still unclear [3]. The polymer rheology is determined to a large extent by the survival of the fraction of initial tube constraints $\Phi(t)$ with time [2]. Creeping motion of the chain from its original tube (reptation), length fluctuations and the removal of constraints by CR determine the amount of the surviving tube fraction. Furthermore, the freed chain sections may dilute the confinement, called dynamic tube dilation [4]. Tube dilation is of particular importance for branched systems undergoing hierarchical relaxation. Dynamic tube dilation $[1,5]$ widens the tube diameter $d$ with time both by dilution with short nonentangled chains (concentration: $1-c$ with $c$ being the volume concentration of long matrix chains) and by dynamic dilation, where $\Phi(t)$ is the fraction of initial constraints that survive at time $t$ :

$$
d(t)=d_{0}[c \Phi(t)]^{-a / 2} .
$$

The (dynamic) dilution exponent $a$ is a fundamental quantity in tube concepts. Accepting this approach dilation and dilution enter models with the same exponent $a$. However, its proper value is largely controversial: e.g., $a=1$ [4,6-10], $a=4 / 3$ [11-13], and $a=2[14,15]$. This controversy is even more important, since the exponent $a$ is related to the fundamental question of the nature of an entanglement. A number of essentially different approaches have been brought forward: (i) Packing models [14,15] consider that an entanglement is made from a given number of strands in an entanglement volume $\simeq d^{3}$ leading to $a=2$; (ii) Binary contact models [16] state that an entanglement is made up by a fixed number of binary interchain contacts $(a=1)$; (iii) Colby and Rubinstein [11] promoted the idea of a fixed number of binary interchain contacts in an entanglement volume $d^{3}$, thereby interrelating the two first models $(a=4 / 3)$; (iv) Novel topological [17] approaches investigate knot invariant polynomials on large rings, where the topology is fixed and relate entanglements with equilibrated topological state statistics resulting in $a=1$; (v) Simulation by tube shrinking algorithms convert 
equilibrated polymer configurations into networks of piecewise linear segments between mutual contact points capturing the impenetrability of chains but deform chains in an ad hoc manner: The results agree with packing models for homopolymer melts $[18,19]$;

Experimentally, the dilation exponent $a$ is very difficult to access; in rheological models the tube size is determined by adjusting its value to reach the best agreement between tube based rheological models and experimental data $[10,20]$. Rheological results on hierarchical branched polymers find exponents $1 \leq a \leq 4 / 3$ [5]. Very recently Watanabe and van Ruymbeke [9] considered initially at early times always $a=1$ and postulated an additional relaxation process (tension reequilibration), in particular important for long-short binary blends due to enhanced fluctuations associated with CR. Exploiting the equivalence of dynamic dilation and dilution by oligomers, in this letter we present a direct measurement of the tube diameter of highly entangled polymers which provides an unambiguous determination of the dilution exponent $a$ on the spatial and temporal scale of the tube. This evidence is supported by results from rheological measurements. Performing neutron spin echo (NSE) measurements on a series of high molecular weight PEO chains diluted up to $50 \%$ by volume (resulting in at least 38 entanglements), we found that the dilution exponent amounts to $a=1.34 \pm 0.04$ resolving thereby the controversy about its value by a view from the microscopic scale both in space and time and confirming the entanglement model of Colby and Rubinstein at the same time. The small amplitude oscillatory shear experiments (SAOS) agree with this result. The dynamic structure factor $S(Q, t)$ from a single protonated chain in a deuterated environment offers direct access to the tube size on nanoscopic length scales. We have

$$
\begin{array}{rr}
\frac{S(Q, t)}{S(Q)}=\left[1-\exp \left(-(Q d)^{2} / 36\right)\right] & S^{\mathrm{loc}}(Q, t) \\
+\exp \left(-(Q d)^{2} / 36\right) & S^{\operatorname{esc}}(Q, t),
\end{array}
$$

where $S^{\text {loc }}(Q, t)$ and $S^{\text {esc }}(Q, t)$ are the contributions from the longitudinal relaxation of Rouse modes (local reptation) along the tube and the escape from the tube, respectively. For long well-entangled chains within the NSE time window $\left[S^{\text {esc }}(Q, t) \simeq 1\right]$ the tube diameter limits the decay of $S(Q, t)$ with time to $Q$-dependent slightly sloping plateaus. The contribution of local reptation is given by

$$
S^{\text {loc }}(Q, t)=\exp \left(t / \tau_{0}\right) \operatorname{erfc}\left(\sqrt{t / \tau_{0}}\right),
$$

with $\tau_{0}=36 /\left(W l^{4} Q^{4}\right)$ being the timescale for the Rouse segment motion along the tube, with $Q$ the momentum transfer during scattering, $W$ the monomeric relaxation length, and $l$ the statistical monomer length. The directly measurable combination $W l^{4}$ is commonly denoted as the Rouse rate. $S(Q, t)$ allows us to measure directly the topological confinement corresponding to the relaxed modulus in rheology; fits are very robust and are only weakly influenced by the detailed value of the Rouse rate or whether or not CLF of the long chains are considered (see Supplemental Material [21]). Measurements at different $Q$ values display a characteristic $Q$ dependence and identify the localization length scale $(d)$. Within the time frame of NSE ( $t \leq 600 \mathrm{~ns})$ for our chains $\Phi(t) \simeq 1$, the dilution effects are solely caused by the concentration of short chains $(1-c)$. Finally, we note that the theory for $S(Q, t)$ does not describe properly the initial Rouse dynamics at early times. Therefore, in this time regime separate fits of the Rouse model [22] are needed to determine $W l^{4}$ (see Supplemental Material [21]). Nearly monodisperse hydrogenous (hPEO-190K) and deuterated (dPEO-190K) linear polyethylene oxide as well as deuterated PEO oligomers (dPEO-1K) as diluent were synthesized by anionic ring opening polymerization. The molecular weights were revealed by SEC/LS (hPEO-190K, dPEO-190K) in combination with conventional PEO calibration and NMR end group analysis (dPEO-1K). For the deuterated linear polymers molecular weights of $M_{n}=190000 \mathrm{~g} / \mathrm{mol}$ (dPEO-190K) and $M_{n}=1330 \mathrm{~g} / \mathrm{mol}(\mathrm{dPEO}-1 \mathrm{~K})$ were obtained. For the hydrogenous PEO (hPEO-190K) as well $M_{n}=190000 \mathrm{~g} / \mathrm{mol}$ was obtained which corresponds to $Z=95$ entanglements per chain (entanglement molecular weight of $M_{e}=2000 \mathrm{~g} / \mathrm{mol}$ yields to $N_{e}=45$ monomer units [23]). SEC revealed polydispersity indices of $M_{w} / M_{n}=1.04$ for hPEO-190K and dPEO-190K and $M_{w} / M_{n}=1.06$ for dPEO-1K. We note that even at $50 \%$ dilution for $a=4 / 3$ or $a=1, Z \simeq 38$ or $Z \simeq 47$, respectively; consequently, the system is still in the strongly entangled regime. Four blends with different volume fractions of short and long chains were prepared in solution and freeze dried from benzene. For all samples the volume fraction of hydrogenous hPEO-190K was fixed to $10 \mathrm{vol} \%$ to provide an appropriate labeling for the neutron scattering experiments. The sample compositions are given in Table I. In the rheological and NSE spectra shown in this Letter, the datasets of the two limiting cases S1-long_100 and S4long_50 are presented, while the corresponding spectra for the other samples as well as the complete synthesis procedure can be found in the Supplemental Material [21].

SANS experiments were performed at KWS2 [24] (MLZ, Munich, Germany), NSE studies at IN15 [25]

TABLE I. Sample compositions.

\begin{tabular}{lccc}
\hline \hline Denomination & hPEO-190K & dPEO-190K & dPEO-1K \\
\hline & [vol \%] & [vol \%] & [vol \%] \\
\hline S1-long_100 & 10 & 90 & 0 \\
S2-long_87.5 & 10 & 77.5 & 12.5 \\
S3-long_75 & 10 & 65 & 25 \\
S4-long_50 & 10 & 40 & 50 \\
\hline \hline
\end{tabular}


(ILL, Grenoble, France) both at $T=413 \mathrm{~K}$. The SAOS investigations were carried out at FORTH (Heraklion, Greece) at $T=353,373,393$, and $413 \mathrm{~K}$, well above the crystallization temperature $T_{c}=341 \mathrm{~K}$ for $\mathrm{S} 1$ and $T_{c}=338 \mathrm{~K}$ for $\mathrm{S} 4$.

The inset of Fig. 1 compares SANS data from the undiluted (S1-long_100) and most diluted samples (S4long_50) with and intensity adjusted to a nominally sample thickness of $1 \mathrm{~mm}$. Both datasets are basically identical and display an unperturbed Gaussian random walk conformation $\left(Q^{-2}\right.$ power law in the higher $Q$ regime) of the long chain component, independent from the presence of oligomeric dilution. They are well described by a random phase approximation (RPA) using Gaussian chain form factors (blue line). At low $Q$ some parasitic forward scattering is observed, as commonly found in the SANS pattern of samples based on deuterated matrices, resulting from air bubbles which exhibit high contrast with the matrix. Figure 1 presents the essential results from the NSE experiments. It displays the single chain dynamic structure factors $S(Q, t) / S(Q, 0)$ for S1-long_100 and S4-long_50 obtained at $\mathrm{T}=413 \mathrm{~K}$ covering a time range up to $530 \mathrm{~ns}$. The time decay at short times mirrors the free Rouse motion

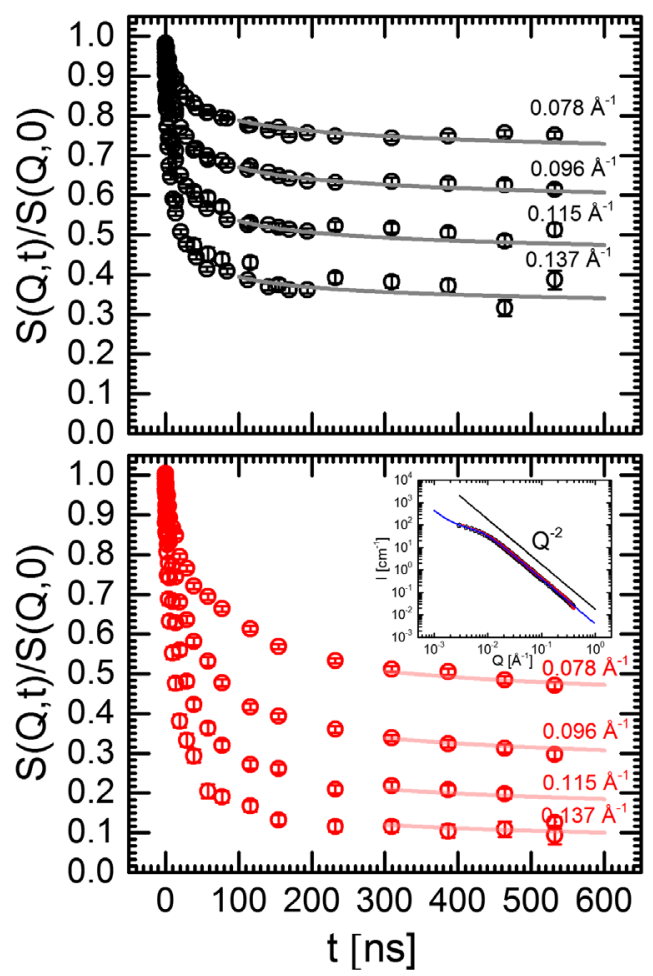

FIG. 1. NSE spectra from the undiluted reference sample (S1long_100, black symbols, upper part) and the most diluted sample (S4-long_50, red symbols, lower part) for different $Q$ at $T=413 \mathrm{~K}$. Lines represent fits with Eq. (2) that reveal $d(c)$. The inset in the lower part shows SANS data from S1-long_100 (black symbols) and S4-long_50 (red symbols). For the blue line see text. within the tube pictured by the Rouse rate $W l^{4}=$ $3 k_{B} T l^{2} / \zeta_{0}$, which is directly related to the monomeric friction coefficient $\zeta_{0}$. Within experimental accuracy we observe a linear increase $W l^{4}$ with increasing dilution according to $W l^{4}(c) \simeq(14890 \pm 1489)[1+(0.76 \pm 0.13) \times$ $(1-c)] \AA^{4} /$ ns. This dependence was extracted by fitting the Rouse model for times $t \leq 5 \mathrm{~ns}$ as discussed in the Supplemental Material [21]. At longer times-well separated from the Rouse regime- the topological confinement due to the entanglements dominates the NSE spectra and pronounced, slightly sloping plateaus, which are characteristic for reptationlike dynamics are observed. The tube diameter was extracted by fitting Eq. (2) to the spectra in the time range $t \geq 100 \mathrm{~ns}$ well beyond the entanglement time $\tau_{e}=\left(N_{e}^{2} l^{4}\right) / \pi^{2} W l^{4}=d^{4} /\left(\pi^{2} W l^{4}\right) \quad$ (S1-long_100, $\tau_{e}=32.9 \mathrm{~ns} ;$ S2-long_87.5, $\tau_{e}=49.1 \mathrm{~ns} ;$ S3-long_75, $\tau_{e}=68.6 \mathrm{~ns}$ ) and $t \geq 300 \mathrm{~ns}$ (S4-long_50, $\tau_{e}=159.5 \mathrm{~ns}$ ).

The solid lines in Fig. 1 show the fitting results based on the following procedure (see also Supplemental Material [21]): The spectral amplitude for each $Q$ has been taken from the short time Rouse fits, where it was considered as a free parameter for each $Q$, while revealing the Rouse rate $W l^{4}$ as a simultaneous fit parameter at the same time. Thus Eq. (2) was fitted to the spectra for each sample for all $Q$ values keeping the tube diameter $d(c)$ as simultaneous and the only free model parameter that allows a determination of $d(c)$ at the scale of the tube with high accuracy. As a result, the $Q$ sequences of the spectra are perfectly reproduced. While the statistical error of the NSE data are negligible small, the systematic error of the fit results is mainly influenced by uncertainties of the Rouse rate that depends on the upper time limit taken into account for the Rouse fits. By varying this limit between $3 \mathrm{~ns}$ and $0.5 \tau_{e}$ the Rouse rate varies by about $10 \%$. As can be seen from Eq. (3) the uncertainty in $W l^{4}$ has an impact on $d(c)$ as well, resulting in a systematic error of about $1 \%$. Thus, directly at the scale of the tube determined tube diameters result to $46.9 \AA$ (S1-long_100), $53.1 \AA$ (S2-long_87.5), $58.8 \AA$ (S3-long_75), and 75.7 $\AA$ (S4-long_50).

Figure 2 presents $d(c)$ as a function of $c$ in a double logarithmic representation. The dynamic dilution exponent follows a power law relation $d(c)=d_{0} c^{-a / 2}$ according to Eq. (1) in excellent agreement as can be seen in Fig. 2. From the slope an exponent $a / 2=0.67$ or $a=1.34$ is obtained, very close to $a=4 / 3$ and clearly distinct from $a=1$.

Figure 3 presents the key rheological results. The inset displays the linear viscoelastic response functions $G^{\prime}(\omega)$ and $G^{\prime \prime}(\omega)$ for the undiluted (S1-long_100) and most diluted (S4-long_50) sample that were obtained by time temperature superposition (TTS) with $T_{\text {ref }}=413 \mathrm{~K}$ (see also Supplemental Material [21]). The temperature dependent density change of the sample material was calculated in accordance to Ref. [26] and used as vertical shift. Afterwards the received spectra were shifted manually 


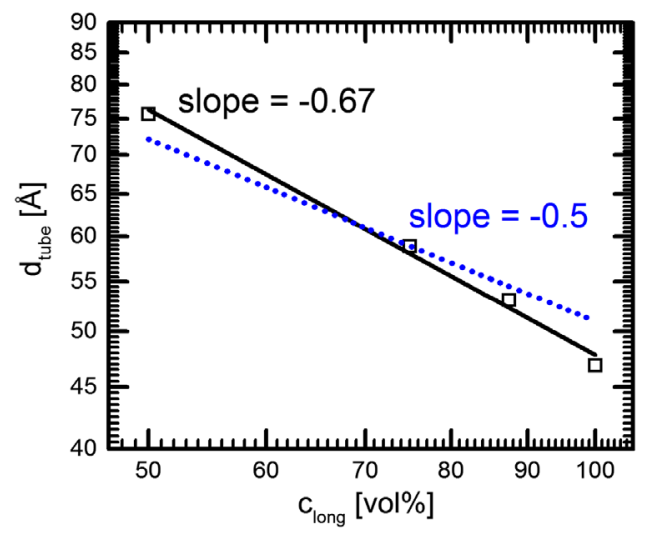

FIG. 2. Double logarithmic representation of the tube diameter $d$ in dependence of oligomeric dilution (symbols). The slope of the fitted power law exponent amounts to -0.67 , revealing a dynamic dilution exponent of $a=1.34$ (black line), close to $a=4 / 3$. For comparison a slope of -0.5 corresponding to $a=1$ (blue dotted line) is shown. Systematic and statistical error bars are within symbol size (see Supplemental Material [21]).

until they overlap [5]. Because of PEO crystallization below $T_{c}=341 \mathrm{~K}$ for $\mathrm{S} 1$ and $T_{c}=338 \mathrm{~K}$ for $\mathrm{S} 4$, the experimentally accessible frequency window is severely restricted. Therefore, the detectable rheological response covers merely the terminal flow regime and the terminal time of the melt, showing the characteristic terminal loss peak of $G^{\prime \prime}(\omega)$, while faster processes such as, e.g., the Rouse relaxation, are not accessible. Predictably, the dilution by oligomers leads to a decrease of the plateau modulus $G_{N}^{0} \propto \rho / d^{2} \propto c / c^{-a}=c^{1+a}$, whith $\rho$ being the polymer density. To quantify this effect $G_{N}^{0} \propto G^{\prime \prime}(\omega)_{\max }$ was determined by using the maximum method which is widely utilized for monodisperse polymer systems in the melt state and in solution as well as for binary mixtures of high molecular weight polymers [27]. To do so $G^{\prime \prime}(\omega)$ was fitted for all samples with the Havriliak-Negami model [28] commonly used for the parametrization of relaxation processes in amorphous systems [29]. Thus the amplitude $G^{\prime \prime}(\omega)_{\max }$ at the peak frequency $\omega_{\max }$ was determined (see inset of Fig. 3). Afterwards the dynamic dilution exponent, linked to the plateau modulus by $G_{N}^{0} \propto G^{\prime \prime}(\omega)_{\max } \propto c / c^{-a}=c^{1+a}$, was extracted by a linear curve fit in double logarithmic representation (Fig. 3). The power law exponent of 2.30 reveals $a=1.30 \pm 0.11$, again close to $a=4 / 3$ and clearly distinct from $a=1$. We note, as shown in the Supplemental Material [21], that the rheological data always reveal a power law exponent between 2.20 and 2.30, no matter which reference temperatures $T_{\text {ref }}=373$ or $T_{\text {ref }}=413 \mathrm{~K}$, nor which method for constructing the master curve [correcting for $\rho(T)$ first and shifting horizontal afterwards or 2-dim residual minimization] is chosen. The same applies if the directly measured and therefore unshifted spectra are analyzed for $\mathrm{T}_{\text {meas }}=$ 353, 373, 393, and $413 \mathrm{~K}$.

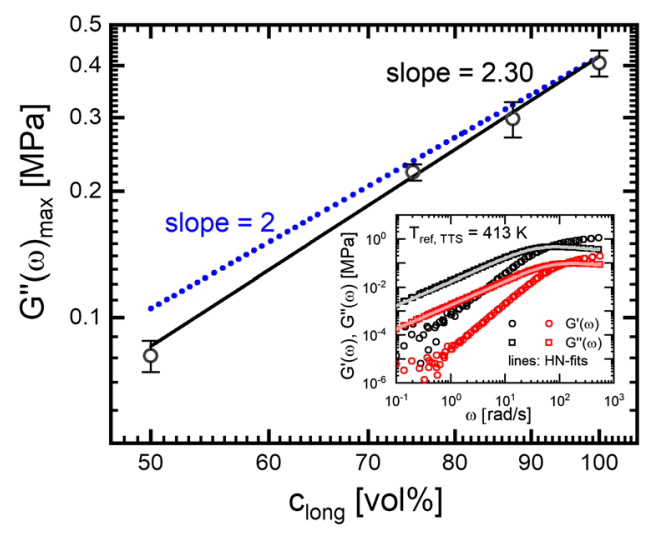

FIG. 3. Double logarithmic presentation of the plateau modulus $G_{N}^{0} \propto G^{\prime \prime}(\omega)_{\max }$ as a function of the matrix chain concentration at $T_{\text {ref }}=413 \mathrm{~K}$. The slope of the power law amounts to $2.30 \pm 0.11$, revealing a dynamic dilution exponent of $a=1.30 \pm 0.11$. For comparison a slope of 2 corresponding to $a=1$ (blue dotted line) is shown. Inset: Storage (circles) and loss modulus (squares) of the undiluted reference (black, S1long_100) and the most diluted sample S4-long_50 (red). The lines represent fits of $G^{\prime \prime}(\omega)$ according to the Havriliak-Negami model used to determine $G^{\prime \prime}(\omega)_{\max }$.

In this work, by applying NSE spectroscopy we measured the dynamic dilution exponent $a$ directly on the scale of the topological constraints, commonly denoted as the tube. The experiments were entirely performed in the highly entangled regime $(Z \geq 40)$. The clear and unambiguous result of $a=1.34 \pm 0.04$ settles a long-standing issue about its proper value and imposes ancillary conditions on rheological theories. Thus, we have replaced the indirect conclusions from adapting theories to polymer melt rheological properties by a direct measurement of dynamic tube dilation-dilution [see Eq. (1)]. A parallel investigation of $G_{N}^{0}(c)$ on the same samples resulted in a dilution exponent compatible with the microscopic result.

A recent rheological study on polymer dilution with oligomers and solvent [20] finds $a$ values between 1 and 1.3 and attributes the larger value 1.3 to be a deviation from the true value 1 caused by CLF/CR relaxation of chains with $Z<20$. The present SAOS investigation yields $a=$ $1.30 \pm 0.11$ from rheology which is compatible with the NSE results and in combination provide unambiguous evidence of the dilution exponent $a$. Furthermore, the microscopic result provides us with the means to select between various models for entanglement formation. It clearly favors the Colby-Rubinstein picture [11]: In order to form an entanglement, a given universal number of binary contacts has to occur within a volume set by the cube of the tube diameter. This requirement determines the tube size $d$ and predicts $a=4 / 3$.

We thank Evelyne van Ruymbeke for enlightening discussions and the EU for partial support (Horizon2020INFRAIA-2016, EUSMI, Project No. 731019). 
b.gold@fz-juelich.de

Present address: ETH Zürich, Department of Materials, Soft Materials Laboratory, Vladimir-Prelog-Weg 5, 8093 Zürich, Switzerland and Paul Scherrer Institut, 5232 Villigen PSI, Switzerland.

[1] T. McLeish, Adv. Phys. 51, 1379 (2002).

[2] M. Doi and S. Edwards, The Theory of Polymer Dynamics (Oxford University Press, Oxford, 1986).

[3] M. Rubinstein and R. Colby, Polymer Physics (Oxford University Press, Oxford, 2003).

[4] G. Marrucci, J. Polym. Sci. 23, 159 (1985).

[5] M. Kapnistos, G. Koutalas, N. Hadjichristidis, J. Roovers, D. J. Lohse, and D. Vlassopoulos, Rheol. Acta 46, 273 (2006).

[6] R. C. Ball and T. C. B. McLeish, Macromolecules 22, 1911 (1989).

[7] S. J. Park and R. G. Larson, Macromolecules 37, 597 (2004).

[8] D. J. Read, K. Jagannathan, S. K. Sukumaran, and D. Auhl, J. Rheol. 56, 823 (2012).

[9] H. Watanabe, Y. Matsumiya, and E. van Ruymbeke, Macromolecules 46, 9296 (2013).

[10] E. van Ruymbeke, V. Shchetnikava, Y. Matsumiya, and H. Watanabe, Macromolecules 47, 7653 (2014).

[11] R. H. Colby and M. Rubinstein, Macromolecules 23, 2753 (1990).

[12] J. H. Lee and L. A. Archer, Macromolecules 35, 6687 (2002).

[13] S. T. Milner and T. C. B. McLeish, Macromolecules 30, 2159 (1997).

[14] Y. H. Lin, Macromolecules 20, 3080 (1987).
[15] L. J. Fetters, D. J. Lohse, D. Richter, T. A. Witten, and A. Zirkel, Macromolecules 27, 4639 (1994).

[16] K. Iwata and S. F. Edwards, J. Chem. Phys. 90, 4567 (1989).

[17] J. Qin and S. T. Milner, Macromolecules 47, 6077 (2014).

[18] R. Everaers, S. K. Sukumaran, G. S. Grest, C. Svaneborg, A. Sivasubramanian, and K. Kremer, Science 303, 823 (2004).

[19] C. Tzoumanekas and D. N. Theodorou, Macromolecules 39, 4592 (2006).

[20] T. Shahid, Q. Huang, F. Oosterlinck, C. Clasen, and E. van Ruymbeke, Soft Matter 13, 269 (2017).

[21] See Supplemental Material at http://link.aps.org/ supplemental/10.1103/PhysRevLett.122.088001 for more details about synthesis, sample preparation, and measurement methods (SANS, NSE, SAOS) as well as a statistical error analysis, which includes Refs. [5,22,24,17].

[22] D. Richter, M. Monkenbusch, A. Arbe, and J. Colmenero, Adv. Polym. Sci. 174, 26 (2005).

[23] L. J. Fetters, D. J. Lohse, S. T. Milner, and W. W. Graessley, Macromolecules 32, 6847 (1999).

[24] H. Maier-Leibnitz Zentrum, J. Large-Scale Res. Facilities 1, A29 (2015).

[25] Institut Laue Langevin, Grenoble, https://doi.ill.fr/10.5291/ ILL-DATA.9-11-1773.

[26] D. I. Sagdeev, M. G. Fomina, and I. M. Abdulagatov, Fluid Phase Equilib. 450, 99 (2017).

[27] C. Liu, J. He, E. van Ruymbeke, R. Keunings, and C. Bailly, Polymer 47, 4461 (2006).

[28] S. Havriliak and S. Negami, Polymer 8, 161 (1967).

[29] F. Kremer and A. Schönhals, Broadband Dielectric Spectroscopy (Springer Verlag, Berlin, Heidelberg, 2003). 